\title{
Collagen Fibrils Appear More Closely Packed in the Prepupillary Cornea: Optical and Biomechanical Implications
}

\author{
Craig Boote, Sally Dennis, Richard H. Newton, Hina Puri, and Keith M. Meek
}

\begin{abstract}
Purpose. The size and organization of stromal collagen fibrils influence the biomechanical and optical properties of the cornea and hence its function. How fibrillar structure varies with position across the cornea has not been fully characterized. The present study was designed to quantify the collagen fibril spacing and diameter across the normal human cornea and to relate this to the properties of the tissue.
\end{abstract}

Methods. Small-angle x-ray diffraction was used to map in detail the variation in fibril spacing and fibril diameter along orthogonal medial-lateral and inferior-superior meridians of five normal human corneoscleral discs.

REsults. Mean fibril diameters remained constant across all corneas up to the limbus, whereupon a sharp increase was observed. However, mean fibril spacing across the central $4 \times$ $3 \mathrm{~mm}$ (prepupillary) cornea measured $5 \%$ to $7 \%$ lower than in the peripheral cornea.

Conclusions. Collagen fibrils in the prepupillary cornea appear to be more closely packed than in the peripheral cornea. Anisotropy in fibril packing across the cornea has potential implications for the transparency and refractive index of the tissue. Biomechanically, it is possible that the higher packing density of stress-bearing collagen fibrils in the prepupillary cornea is necessary for maintaining corneal strength, and hence curvature, in a region of reduced tissue thickness. By inference, these results could have important implications for the development of corneal models for refractive surgery. (Invest Ophthalmol Vis Sci. 2003;44:2941-2948) DOI: 10.1167/iovs.03-0131

B $y$ virtue of its remarkable mechanical strength and transparency, the human cornea serves both as a protective barrier and as the main refractive component of the eye. Conventionally, the central third of the cornea is considered the optical zone ${ }^{1}$ and provides the bulk of the cornea's refractive function, constituting approximately $70 \%$ of the total focusing of the eye. The near-spherical optical zone forms the foveal image through the pupil and is thus often referred to as the prepupillary cornea, whereas the remaining peripheral cornea is less curved and serves mainly as a refractive surface for peripheral vision. The cornea merges with the sclera at the

From the Department of Optometry and Vision Sciences, Cardiff University, Cardiff, Wales, United Kingdom.

Supported by Wellcome Trust Grant WT055710 and Medical Research Council Grant G0001033.

Submitted for publication February 6, 2003; revised March 6, 2003; accepted March 9, 2003.

Disclosure: C. Boote, None; S. Dennis, None; R.H. Newton, None; H. Puri, None; K.M. Meek, None

The publication costs of this article were defrayed in part by page charge payment. This article must therefore be marked "advertisement" in accordance with 18 U.S.C. $\$ 1734$ solely to indicate this fact.

Corresponding author: Craig Boote, Department of Optometry and Vision Sciences, Cardiff University, Redwood Building, Cardiff, Wales CF10 3NB, UK; bootec@cf.ac.uk. narrow annular limbus. The surface zones of the cornea are shown schematically in Figure 1.

Corneal function is governed largely by the structure of the stromal extracellular matrix, the bulk of which in human cornea comprises collagen fibrils arranged in approximately 200 parallel lamellae. ${ }^{2}$ Fibrils within a lamella are parallel to each other and to the corneal surface, but run at angles in relation to fibrils in adjacent lamellae. This collagen network is responsible for the cornea's mechanical strength. When a force is applied to the cornea, the restoring force in the stretched fibrils balances the applied force, and the fibrils confer tensile reinforcement on the tissue. The diameter of collagen fibrils, their orientation in relation to the applied force, and the collagen content of the tissue all determine how effectively the fibrils can reinforce the tissue and hence determine its tensile strength. ${ }^{3,4}$ Tissue strength is paramount to the refractive function of the cornea because of the need for the cornea to maintain correct surface curvature under intraocular pressure. Fibril organization in the stroma is also crucial to corneal transparency. Current theory requires a measure of fibrillar order for corneal light transmission, ${ }^{5}$ and the uniformity of fibril diameters and their packing are thought to be instrumental in the establishment of a transparent cornea. ${ }^{2,5}$

$\mathrm{X}$-ray diffraction is a technique that involves passing an intense beam of $x$-rays through an intact isolated cornea and recording the intensity of the scattered $\mathrm{x}$-rays on a detector placed behind the specimen. Each lamella in the corneal stroma gives rise to small-angle equatorial diffraction due to interference between $\mathrm{x}$-rays scattered from its constituent axially aligned fibrils (Fig. 2). This is because, over a short range, fibrils tend to be of a fairly uniform diameter and regularly spaced in the lateral direction. From the diffraction pattern, we can determine several key features of the cornea's internal structure, including the mean separation of fibrils and their average diameter (see Fig. 2). A comprehensive treatment of the technique can be found in a recent review. ${ }^{6}$

Fibril spacing and diameter in the central cornea have been measured in a number of species, both by small-angle x-ray diffraction ${ }^{7}$ and electron microscopy. ${ }^{8,9,10}$ However, there are only a very limited number of reports in the literature characterizing variations in fibrillar structure as a function of position across the cornea, despite the potential clinical importance of this knowledge. Notably, Borcherding et al. ${ }^{11}$ measured semiquantitatively the variation in fibril diameter and spacing across the human cornea from electron micrographs, reporting that both parameters remained constant with increasing radial position, before increasing rapidly at the limbus. However, measurements from electron microscopy have tended to show considerable variation among studies, ${ }^{8-11}$ which has been attributed largely to tissue shrinkage during specimen preparation. ${ }^{12,13}$ By this token, structural parameters are more readily obtained from scattering experiments, which require no chemical fixing or dehydration of specimens and can thus be performed under conditions more comparable with those encountered in vivo. Furthermore, $\mathrm{x}$-ray data are highly illustrative 


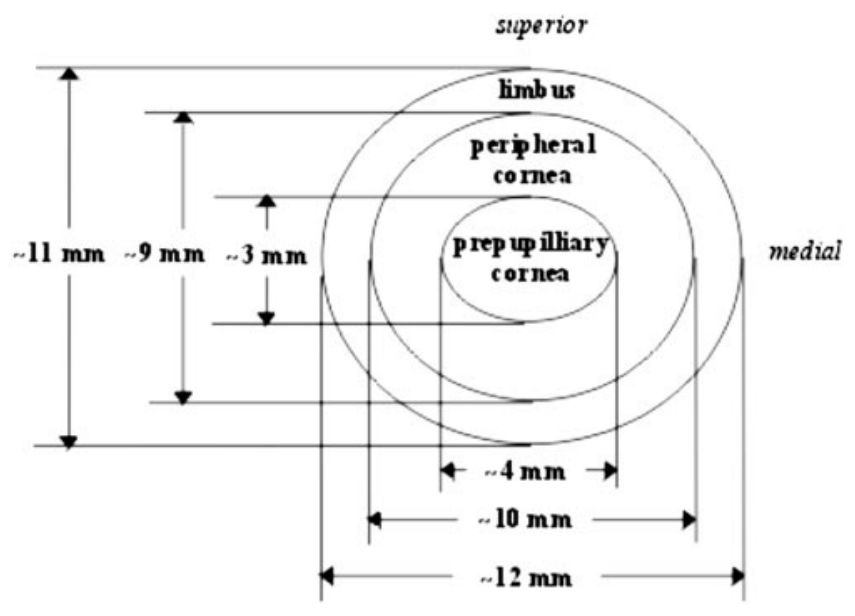

Figure 1. Conventional designation of surface zones in human cornea (anterior corneal face shown).

because they represent an average measurement of the volume of tissue through which the x-ray beam passes, sampling every fibril in the path of the $x$-rays throughout the entire corneal thickness. Recently, Daxer et al. ${ }^{14}$ used x-ray diffraction techniques to study the spatial variation in human corneal fibril diameters and reported no significant differences within a central $7-\mathrm{mm}$ region. In the present study, we built on this work by quantifying in detail the variation in fibril diameters and, moreover, fibril spacings across the human cornea, by using small-angle $\mathrm{x}$-ray diffraction, and we related the findings to the tissue's properties.

\section{Materials ANd Methods}

\section{Specimens}

Five time-expired, whole, excised human corneas, each having $3 \mathrm{~mm}$ of scleral rim attached, were obtained from the UK Corneal Transplant
Service Eye Bank (Bristol, UK). Corneal epithelial and endothelial cell layers were removed before examination with $\mathrm{x}$-rays. Tissue hydration of the specimens (expressed as mass of water/mass of dry cornea) ranged from 2.0 to 4.0. The tenets of the Declaration of Helsinki were observed throughout.

\section{Data Collection}

Small-angle x-ray diffraction patterns were collected on Station 2.1 at the UK Synchrotron Source at Daresbury, using a 9-m-long camera equipped with a multiwire gas detector. The $\mathrm{x}$-ray beam had a wavelength of $0.154 \mathrm{~nm}$ and a cross section at the specimen measuring 0.5 $\mathrm{mm}$ vertically and $2 \mathrm{~mm}$ horizontally. Specimens were placed in airtight Perspex (Databank, UK) chambers with Mylar (DuPont-Teijin, UK) windows to minimize tissue dehydration. Incident $\mathrm{x}$-rays were passed through the anterior corneal face parallel to the optical axis. Diffraction patterns were recorded at 0.4 - to $1.0-\mathrm{mm}$ intervals along the orthogonal inferior-superior and medial-lateral corneal meridians, using a stepper motor system to translate the specimen in the vertical direction, the sample being rotated through $90^{\circ}$ between transects. Exposure time per data point was 3 minutes.

\section{Data Processing}

Figure 3 shows a typical $x$-ray diffraction pattern from the center of one of our specimens. X-ray intensity profiles were measured along a vertical transect through the center of each pattern, as shown in the figure, because this was the direction in which the $\mathrm{x}$-ray beam was most finely focused. A typical resultant profile is shown in Figure 4a. Data processing then proceeded in 4 steps:

1. A square-power law background curve was fitted to and subtracted from the experimental data (Fig. 4a). This removed scattering from stromal matrix components other than fibrillar collagen

2. Assuming cylindrical collagen fibrils, small-angle equatorial $\mathrm{x}$ ray diffraction from a corneal lamella can be approximated as the fibril transform (i.e., scattering from a single fibril), multiplied by the interference function (deriving from the ordered arrangement of the cylinders). ${ }^{15,16} \mathrm{~A}$ theoretical fibril transform was fitted to the experimental data by varying two parameters:

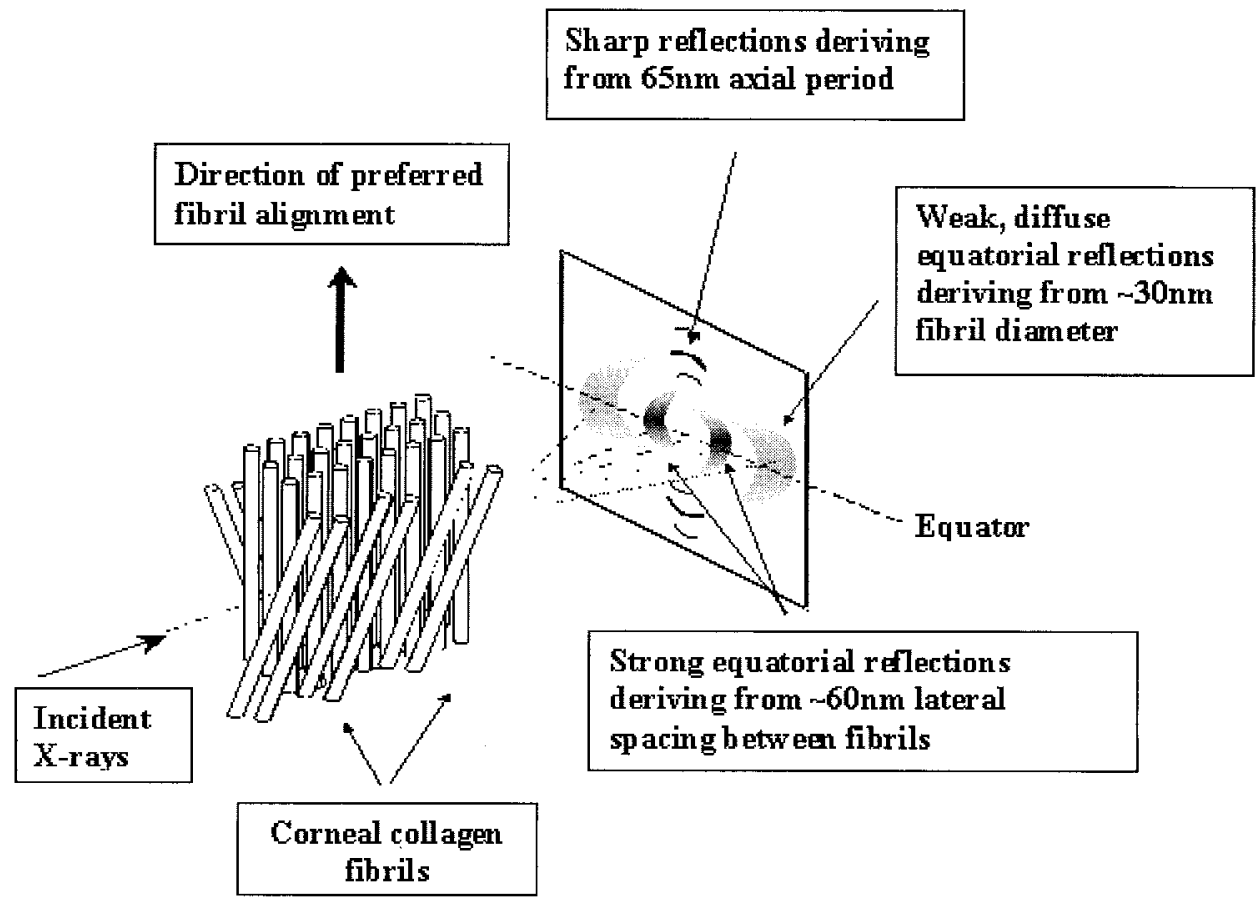

FIGURE 2. Small-angle $\mathrm{x}$-ray diffraction from corneal collagen fibrils. 


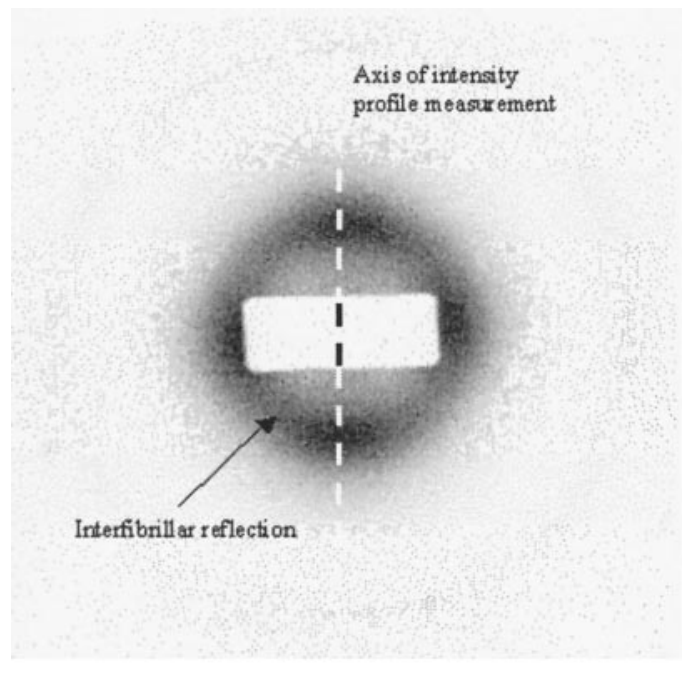

(a)

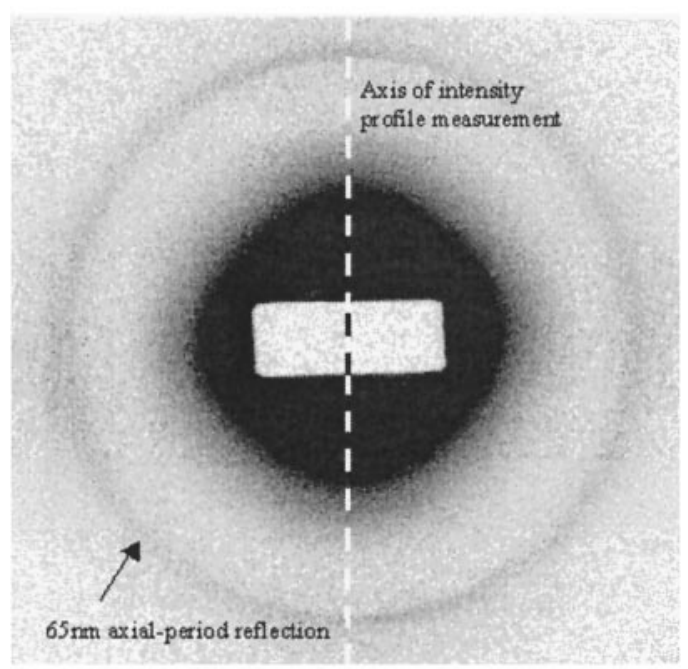

(b)

FiguRe 3. A typical small-angle $x$-ray diffraction pattern from the center of human cornea. The pattern is shown at two different display thresholds to highlight (a) the equatorial interfibrillar reflection and (b) the meridional axial period reflection. Intensity profiles were measured along a vertical transect through the pattern center, as indicated.

the fibril radius and an arbitrary scaling factor (Fig. 4b). Fitting was performed on the first subsidiary maximum of the experimental data (Fig. 4b), because this peak derives entirely from the fibril transform, with no significant contribution from the interference function. ${ }^{16}$ The experimental data were then divided point-for-point by the calculated fibril transform to leave the interference function, whose peak position is governed by the average interfibrillar Bragg spacing (Fig. 4c).

3. The interfibrillar Bragg spacing and fibril radius were calibrated from the position of the $67-\mathrm{nm}$ meridional reflection from a diffraction pattern of hydrated rat tail tendon.

4. The average center-to-center interfibrillar spacing $(i)$ was calculated from the interfibrillar Bragg spacing $(p)$ with $i=1.12 p$, where 1.12 is a packing factor that assumes that the arrangement of fibrils in a lamella approximates the short-range order of a liquid. ${ }^{16}$

\section{Results}

Fibril diameters along superior-inferior and medial-lateral meridians of five human corneas are shown in Figure 5. The results show that the fibril diameter remained constant from the corneal center up to the limbus in all five corneas, with diameters typically measuring 32 to $34 \mathrm{~nm}$. This is consistent with previous work ${ }^{11,14}$. In agreement with the Borcherding study, ${ }^{11}$ fibril diameter rose sharply across the limbus, increasing by up to approximately $75 \%$. There was no evidence of any dependency of these results on tissue hydration, an observation that is consistent with the findings of Daxer et al. ${ }^{14}$
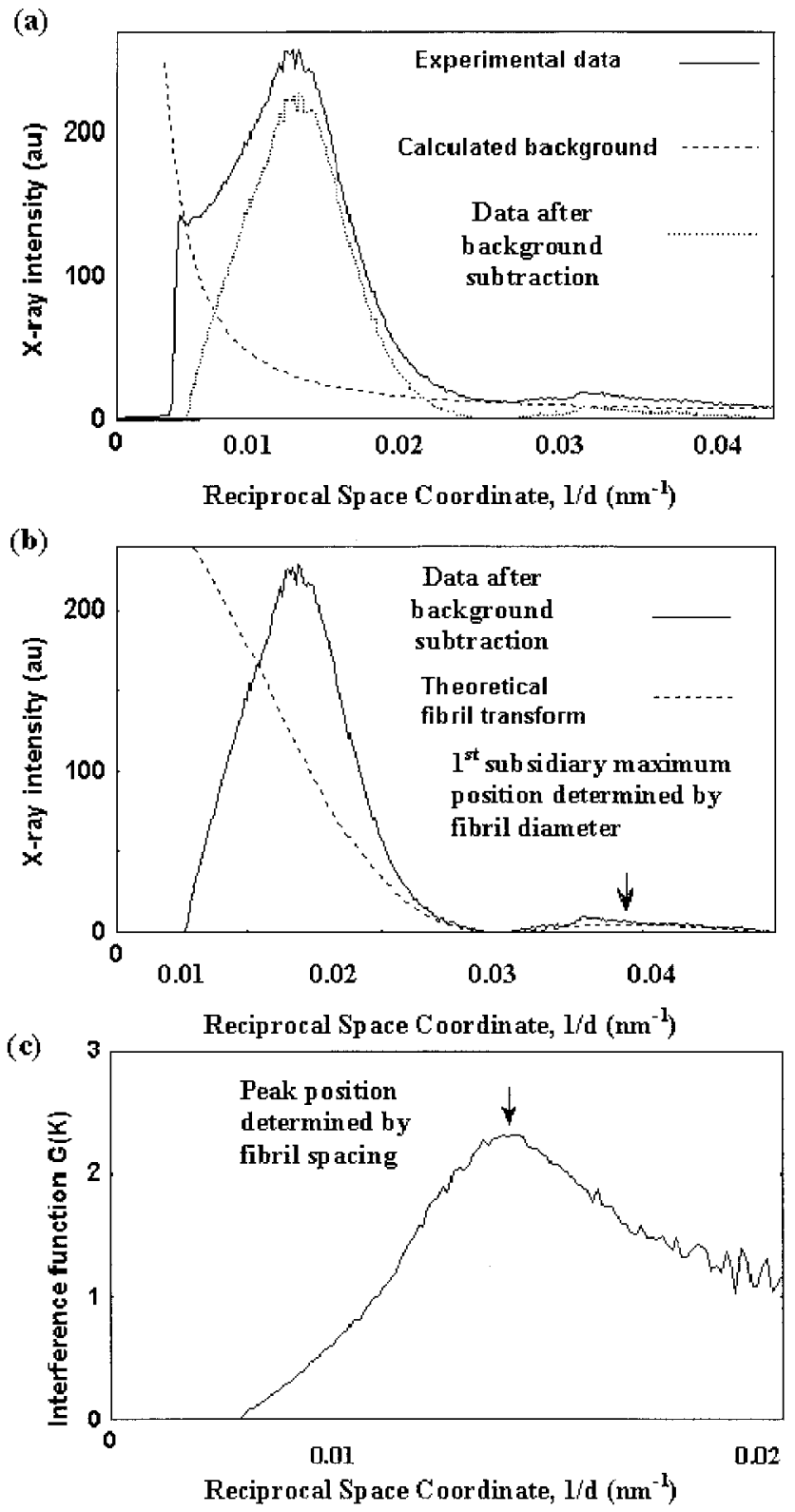

FiguRe 4. Processing of small-angle corneal diffraction data, showing (a) fitting of a square-power law background to the experimental intensity profile and (b) fitting of a theoretical fibril transform to the background-subtracted data. The position of the first subsidiary maximum peak is determined by the fibril diameter. (c) Dividing the experimental data point-for-point by the fibril transform yields the interference function, the peak position of which is determined by the interfibrillar spacing. 

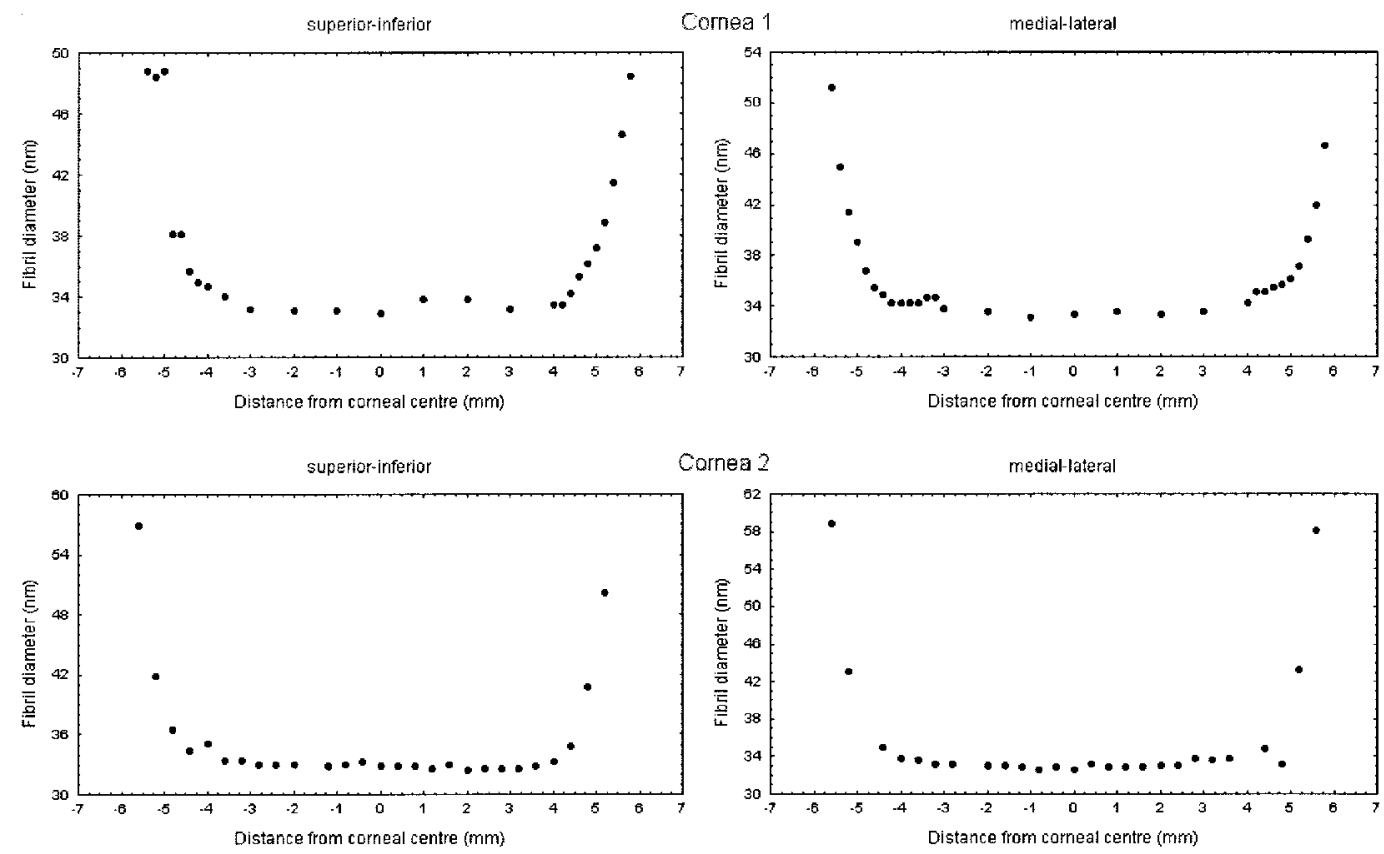

Cornea 2 medial-lateral
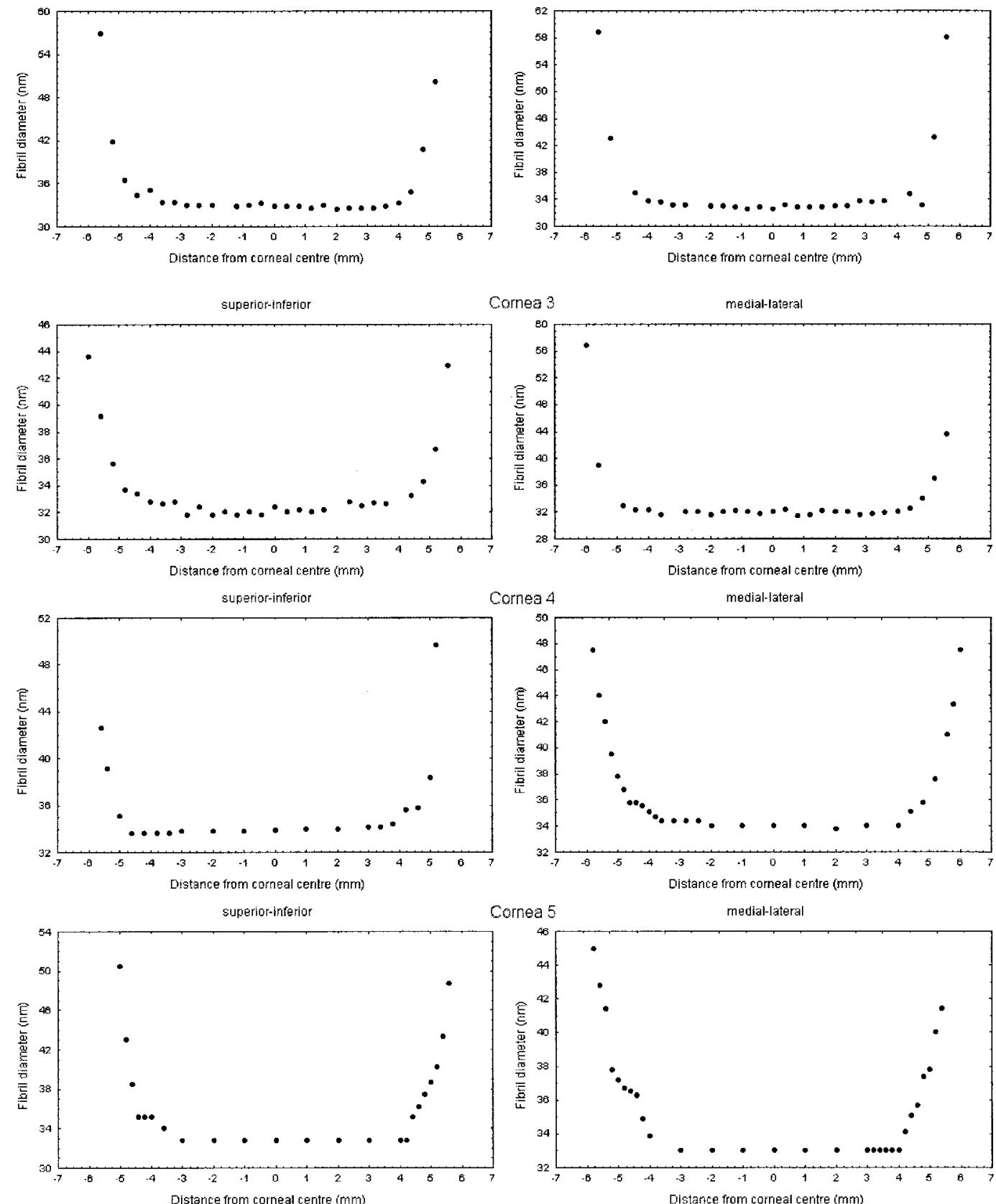

Conea 5

medial-lateral

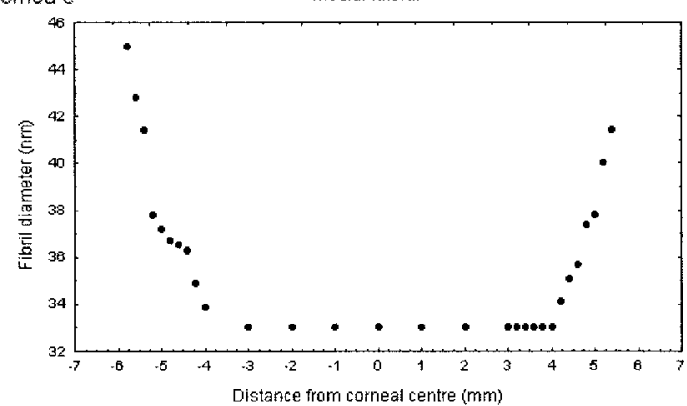

FIGURE 5. Fibril diameters across orthogonal superior-inferior and medial-lateral meridians of five normal human corneas. 

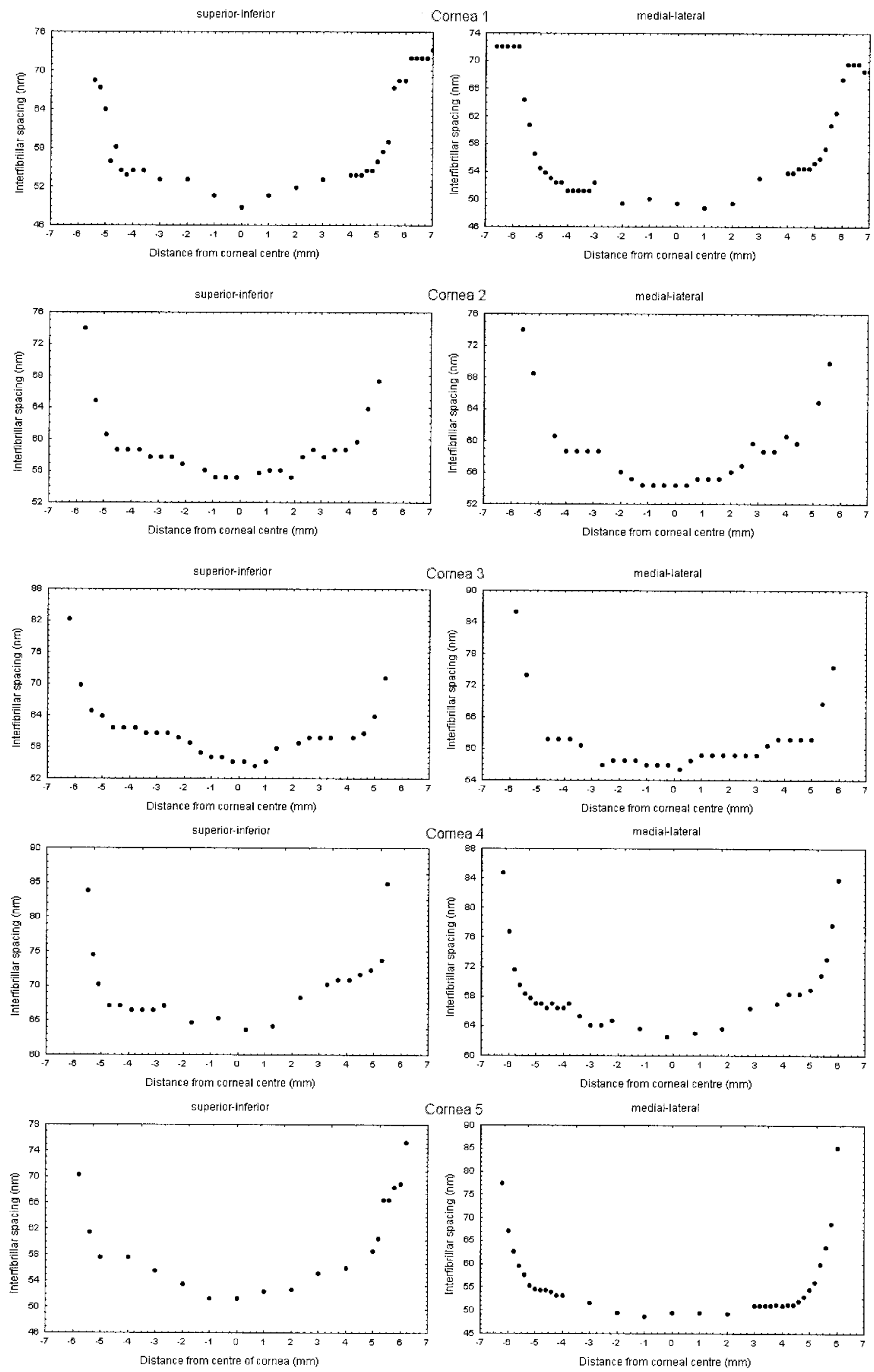

FIGURE 6. Center-to-center interfibrillar spacing across orthogonal superior-inferior and medial-lateral meridians of five normal human corneas. 

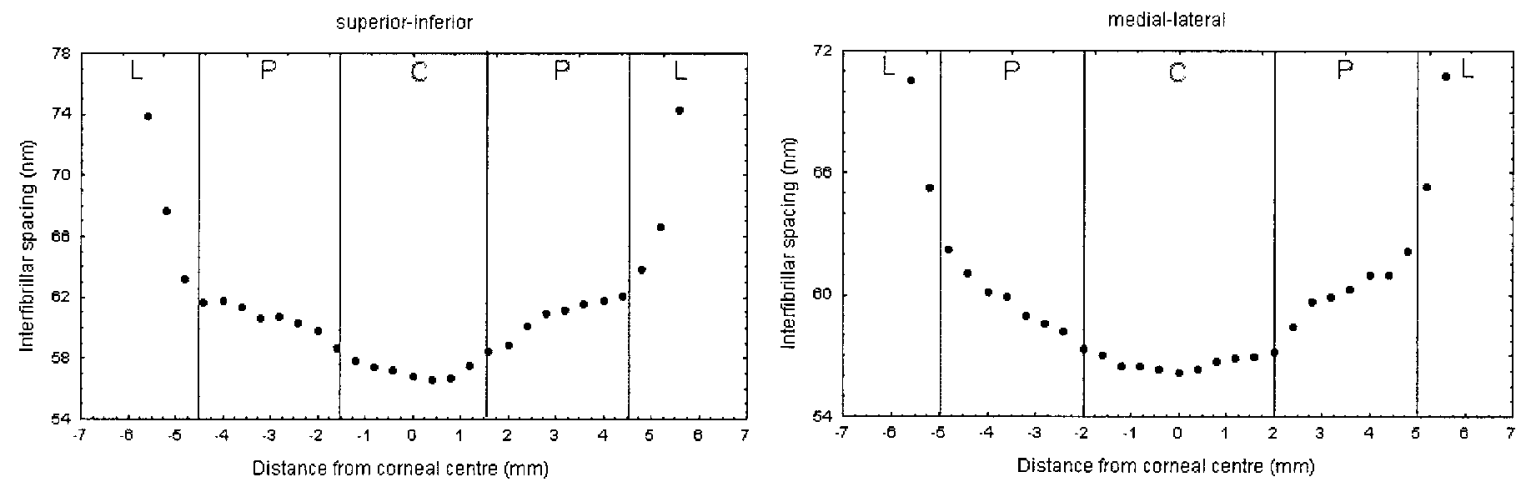

FIGURE 7. Center-to-center interfibrillar spacing, averaged over five corneas and normalized to physiological hydration. Central (prepupillary), peripheral, and limbal corneal surface zones are denoted by $\mathrm{C}, \mathrm{P}$, and L, respectively.

Figure 6 shows the equivalent data for the center-to-center interfibrillar spacing. In all five specimens the average fibril spacing was clearly reduced near the corneal center compared with the more peripheral regions, a result that contrasts with previous measurements from electron microscopy. ${ }^{11}$ Across the limbus, fibril spacing rapidly increased by approximately $25 \%$ to $40 \%$, an observation that is consistent with previous findings. ${ }^{11}$

Figure 7 shows interfibrillar spacing averaged over the five corneas and normalized to physiological hydration, onto which corneal surface zone boundaries have been superimposed. The mean fibril spacing within the prepupillary and peripheral corneal zones may be compared by inspection of the data in Table 1. Prepupillary fibril spacing measured 5\% to $7 \%$ lower than in the peripheral cornea. A two-tailed Student's $t$-test revealed this difference to be statistically significant $(P \ll$ 0.01 ) for all five specimens (Table 1). In contrast, reference to Table 2 shows that no significant difference was found in the fibril diameter measurements between the two corneal zones $(P \geq 0.01)$. In addition, fibril spacing for the five individual corneas (normalized to physiological hydration) were grouped and analyzed using a two-tailed $t$-test. This again disclosed a significant difference between the two corneal zones $(P=1.1$ $\left.\times 10^{-14}\right)$.

The variation in fibril spacing across the cornea appears to be qualitatively unaffected by tissue hydration. Reference to Table 1 shows that the differences in hydration $(H)$ between specimens affected the fibril spacing in all corneal zones uniformly. The data correlate well with data in previous $\mathrm{x}$-ray diffraction studies of stromal hydration. ${ }^{17}$

TABLE 1. Comparison of Interfibrillar Spacing in the Prepupilliary and Peripheral Zones of Five Human Corneas

\begin{tabular}{|c|c|c|c|c|}
\hline Cornea & Hydration & $\begin{array}{c}\text { Corneal } \\
\text { Zone }\end{array}$ & $\begin{array}{c}\text { Mean } \\
\text { Interfibrillar } \\
\text { Spacing (nm) }\end{array}$ & $P(=)$ \\
\hline \multirow[t]{2}{*}{1} & 2.0 & Prepupilliary & $49.7 \pm 1.2$ & $2.7 \times 10^{-9}$ \\
\hline & & Peripheral & $53.4 \pm 2.3$ & \\
\hline \multirow[t]{2}{*}{2} & 2.7 & Prepupilliary & $55.2 \pm 0.6$ & $1.9 \times 10^{-13}$ \\
\hline & & Peripheral & $58.5 \pm 1.5$ & \\
\hline \multirow[t]{2}{*}{3} & 2.9 & Prepupilliary & $56.7 \pm 1.4$ & $7.4 \times 10^{-10}$ \\
\hline & & Peripheral & $60.5 \pm 2.2$ & \\
\hline \multirow[t]{2}{*}{4} & 4.0 & Prepupilliary & $63.7 \pm 0.9$ & $6.7 \times 10^{-5}$ \\
\hline & & Peripheral & $67.0 \pm 1.9$ & \\
\hline \multirow[t]{2}{*}{5} & 2.0 & Prepupilliary & $50.0 \pm 1.2$ & $3.0 \times 10^{-4}$ \\
\hline & & Peripheral & $53.2 \pm 1.8$ & \\
\hline
\end{tabular}

Data are means \pm SD. The statistical significance level of the difference between the zones is shown.

\section{DisCussion}

Our results disclose that fibril packing is nonuniform over the corneal surface and point to a more compact fibril matrix in the prepupillary cornea. Mean center-to-center interfibrillar separation measured $5 \%$ to $7 \%$ lower in the prepupillary cornea compared with the peripheral, a result that proved significant after statistical testing (Table 1). Considering the distinct functionality of the prepupillary and peripheral cornea and the well-established link between corneal function and fibril organization, it would be surprising if our results held no functional significance. In seeking an explanation for the results, it is instructive to consider the potential influence of fibril compaction on three corneal properties crucial to its function: transparency, refractive index, and mechanical strength.

The two most well-established models for describing the dependency of corneal transparency on fibril ultrastructure are those of Hart and Farrell ${ }^{18}$ and Freund et al. ${ }^{19}$ Although these models make considerable assumptions and simplifications in describing the structure of the stroma, they have been useful for assessing the importance of structural parameters on lightscattering in the cornea. It is implicit in both models that increased packing density of collagen fibrils predicts a reduction in tissue transparency. ${ }^{18,19}$ Thus, the presence of more closely packed fibrils centrally seems detrimental to the refractive function of the most optically important corneal region. However, care should be taken in interpreting the results of transparency models, because the parameters included in the models are often mutually dependent. ${ }^{18,19}$ Moreover, corneal

TABle 2. Comparison of Fibril Diameter in the Prepupilliary and Peripheral Zones of Five Human Corneas

\begin{tabular}{|c|c|c|c|c|}
\hline Cornea & Hydration & $\begin{array}{c}\text { Corneal } \\
\text { Zone }\end{array}$ & $\begin{array}{l}\text { Mean Fibril } \\
\text { Diameter } \\
\text { (nm) }\end{array}$ & $P(=)$ \\
\hline \multirow[t]{2}{*}{1} & 2.0 & Prepupilliary & $33.4 \pm 0.6$ & 0.01 \\
\hline & & Peripheral & $34.3 \pm 1.2$ & \\
\hline \multirow[t]{2}{*}{2} & 2.7 & Prepupilliary & $33.0 \pm 0.3$ & 0.01 \\
\hline & & Peripheral & $33.5 \pm 1.1$ & \\
\hline \multirow[t]{2}{*}{3} & 2.9 & Prepupilliary & $32.0 \pm 0.4$ & 0.01 \\
\hline & & Peripheral & $32.4 \pm 0.8$ & \\
\hline \multirow[t]{2}{*}{4} & 4.0 & Prepupilliary & $34.0 \pm 0.1$ & 0.06 \\
\hline & & Peripheral & $34.6 \pm 0.9$ & \\
\hline \multirow[t]{2}{*}{5} & 2.0 & Prepupilliary & $33.0 \pm 0.1$ & 0.05 \\
\hline & & Peripheral & $33.9 \pm 1.3$ & \\
\hline
\end{tabular}

Data are means $\pm \mathrm{SD}$. The statistical significance level of the difference between the zones is also shown. 
thickness is at least 20\% lower in the center of the cornea than at the periphery. ${ }^{20,21}$ In terms of transparency, a thinner prepupillary cornea tends to compensate for a higher fibril packing density.

The issue of the cornea's refractive index is clearer. Bearing in mind that fibril diameters remain constant across the cornea (Fig. 5), we deduce that smaller center-to-center fibril spaces result in a higher collagen fibril volume fraction. Given that stromal collagen fibrils have a higher refractive index than the intervening material, ${ }^{22}$ it follows that closer packing of fibrils predicts a higher refractive index, and hence dioptric power, in the tissue. In view of our findings, there is clearly room for more detailed study into the variation of refractive index and transparency as a function of position in the cornea.

What of the potential impact of our results on the biomechanics of the cornea? The cornea is reinforced by collagen fibrils, as described earlier. These fibrils are strongest axially, and directions of preferred fibril orientation thus associate with directions of heightened tissue strength. Fibril diameters are also biomechanically important, because they determine the fibrils critical length, ${ }^{3}\left(l_{\mathrm{c}}\right)$ given by

$$
l_{\mathrm{c}}=d \sigma_{\mathrm{f}} / 2 \tau
$$

where $d$ is the fibril diameter, $\sigma_{f}$ is the fibril's tensile strength and $\tau$ is the shear stress exerted on the fibril by the ground substance (we define ground substance as being stromal matrix elements other than fibrillar collagen). The critical length is the minimum fibril length required for effective tissue reinforcement. ${ }^{3}$ As long as this condition is met, the tensile strength of the tissue $\left(\sigma_{t}\right)$ is determined by the volume fraction of collagen present $(\beta)$

$$
\sigma_{\mathrm{t}}=\beta \sigma_{\mathrm{f}}+(1-\beta) \sigma_{\mathrm{g}}
$$

where $\sigma_{\mathrm{f}}$ and $\sigma_{\mathrm{g}}$ are the tensile strengths of the fibrils and ground substance, respectively. ${ }^{3,23}$ It is our hypothesis that the higher packing density of collagen fibrils we have observed in the prepupillary cornea is necessary to maintain tissue strength, bearing in mind that the cornea is thinner centrally. ${ }^{20,21}$ Inspection of equation 2 reveals that, for $\sigma_{\mathrm{f}}>$ $\sigma_{\mathrm{g}}$, increasing the volume fraction of collagen produces a proportional increase in the mechanical strength of the tissue. Hence, we expect reduced prepupillary fibril spacing and thus increased collagen volume fraction to result in a stronger central cornea. Such a mechanism could help to preserve dioptric stability in the cornea by helping to maintain surface curvature in the presence of variations in tissue thickness. Of course, we are assuming that corneal collagen fibrils are at least as long as their critical length. Although the exact length of collagen fibrils in the cornea is unknown, Maurice $^{24}$ observed that corneal lamellae appear to run uninterrupted from limbus to limbus. Furthermore, electron microscopy has indicated that the critical length condition is met by most of the stress-bearing collagen fibrils in other connective tissues. ${ }^{25}$

We can currently only speculate as to the mechanisms that could be driving the changes in fibril spacing across the cornea. However, one possibility is that it may be related to variations in hydration across the tissue. Fibril spacing in the cornea is known to be highly sensitive to the tissue's water content. Reference to previous $\mathrm{x}$-ray scattering work on corneal stroma reveals that, at the hydration levels encountered in our work, water is exclusively deposited into or removed from the interfibrillar spaces, rather than within the fibrils themselves. ${ }^{17,26}$ Under these condi- tions, therefore, even subtle variations in tissue hydration could be expected to produce changes in the spacing of the fibrils, without affecting their diameter. How tissue hydration varies across the cornea is currently unknown, and clearly there is a need for detailed investigation of this question.

Information about the tissue strength of the cornea as a function of position and the parameters that influence it have obvious clinical relevance. Current models used in the simulation of refractive surgery make complex assumptions regarding the ultrastructure of the corneal stroma, ${ }^{27,28}$ because there is a general lack of detailed structural information. Recently, scattering methods have been used in attempts to characterize tissue structure. Newton and Meek $^{29,30}$ determined the variation in collagen fibril orientation across the cornea and reported a gradual alteration in the preferred fibril orientation, from orthogonal at the corneal center to circumferential at the limbus. Such anisotropy in the cornea could explain why surgical incisions at some positions in the cornea are more likely to induce astigmatism than at others. ${ }^{31}$ There appears to be no obvious correlation between our data and the variation of fibril orientations across the cornea. Nevertheless, our contention is that anisotropy in fibril packing across the cornea may well have similarly important clinical implications because of the influence of collagen packing density on tissue strength and hence corneal curvature.

We have presented herein the first evidence that fibrils are more closely packed in the optical zone of human cornea and argue that this could have important implications for the properties, and hence the function, of the cornea. So far, we have collected detailed structural data covering the mediallateral and inferior-superior corneal meridians. We propose to compose a map of structural parameters over the entire corneal surface at similarly high resolution in the future. It is possible that detailed mapping of structural parameters across the corneal surface may be highly beneficial in the development of corneal models to optimize refractive surgery.

\section{Acknowledgments}

The authors thank Val Smith of the UK Corneal Transplant Service Eye Bank (Bristol, UK) and Gunter Grossmann and the staff of the Council for the Central Laboratory of the Research Councils Synchrotron Radiation Source (Daresbury, UK).

\section{References}

1. Waring GO. Making sense of keratospeak II: proposed conventional terminology for corneal topography. Refract Corn Surg. 1989;5:362-367.

2. Maurice DM. The structure and transparency of the corneal stroma. J Physiol. 1957;136:263-286.

3. Hukins DWL, Aspden RM. Composition and properties of connective tissues. Trends Biochem Sci. 1985;10:260-264.

4. Jeronimidis G, Vincent JFV. Composite materials. In: Hukins DWL ed. Connective Tissue Matrix. Macmillan; 1984:187-210.

5. Farrell RA. Corneal transparency. In: Albert DM, Jacobiec SA, eds. Principles and Practice of Opbthalmology. Philadelphia: Saunders; 1994.

6. Meek KM, Quantock AJ. The use of X-ray scattering techniques to determine corneal ultrastructure. Prog Retinal Eye Res. 2001;20: 95-137.

7. Meek KM, Leonard DW. Ultrastructure of the corneal stroma: a comparative study. Biophys J. 1993;64:273-280.

8. Freund DE, McCally RL, Farrell RA, Cristol SM, L'Hernault NL, Edelhauser HF. Ultrastructure in anterior and posterior stroma of 
perfused human and rabbit corneas: relation to transparency. Invest Ophthalmol Vis Sci. 1995;36:1508-1523.

9. Kanai A, Kaufman HE. Electron microscopic studies of corneal stroma: ageing changes of collagen fibres. Ann Ophthalmol. 1973; 5:285-292.

10. Craig AS, Parry DAD. Collagen fibrils of the vertebrate corneal stroma. J Ultra Mol Struct Res. 1981;74:232-239.

11. Borcherding MS, Blacik LJ, Sittig RA, Bizzell JW, Breen M, Weinstein HG. Proteoglycans and collagen fibre organisation in human corneoscleral tissue. Exp Eye Res. 1975;21:59-70.

12. Fullwood NJ, Meek KM. A synchrotron X-ray study of the changes occurring in the corneal stroma during processing for electronmicroscopy. J Microsc. 1993;169:53-60.

13. Craig AS, Robertson JG, Parry DAD. Preservation of corneal collagen fibril structure using low-temperature procedures for electron microscopy. J Ultra Mol Struct Res. 1986;96:172-175

14. Daxer A, Misof K, Grabner B, Ettl A, Fratzl P. Collagen fibrils in the human corneal stroma: structure and ageing. Invest Ophthalmol Vis Sci. 1998;39:644-648.

15. Oster G, Riley DP. Scattering from cylindrically symmetric systems. Acta Crystallogr. 1952;5:272-276.

16. Worthington CR, Inouye H. X-ray diffraction study of the cornea. Int J Biol Macromol. 1985;7:2-8.

17. Meek KM, Fullwood NJ, Cooke PH, et al. Synchrotron X-ray diffraction studies of the cornea, with implications for stromal hydration. Biophys J. 1991;60:467-474.

18. Hart RW, Farrell RA. Light scattering in the cornea. J Opt Soc Am. 1969;59:766-774.

19. Freund DE, McCally RL, Farrell RA. Direct summation of fields for light scattering by fibrils with applications to normal corneas. $A p p l$ Opt. 1986;25:2739-2746.
20. Martola EL, Baum JL. Central and peripheral corneal thickness. Arch Ophthalmol. 1968;79:28-30.

21. Edmund C. Determination of the corneal thickness profile by optical pachometry. Acta Ophthalmol. 1987;65:147152

22. Leonard DW, Meek KM. Estimation of the refractive indices of collagen fibrils and ground substance of the corneal stroma using data from X-ray diffraction. Biophys J. 1997;72:13821387.

23. Krenchel H. Fibre Reinforcement. Copenhagen, Denmark: Akademisk Forlag; 1964.

24. Maurice DM. The cornea and sclera. In: Davson H, ed. The Eye. New York: Academic Press; 1969:489-599.

25. Muir H, Bullough P, Maroudas A. The distribution of collagen in human articular cartilage with some of its physiological implications. J Bone Surg Ser B. 1970;52:554-563.

26. Fratzl P, Daxer A. Structural transformation of collagen fibrils in corneal stroma during drying: an X-ray scattering study. Biophys J. 1993;64:1210-1214.

27. Hanna KD, Jouve FE, Waring GO, Ciarlet PG. Computer simulation of arcuate keratotomy for astigmatism. Refract Corneal Surg. 1992;8:152-163.

28. Moreira H, Campos M, Sawush MR, McDonnell JM, Sand B, McDonald PJ. Holmium laser thermokeratoplasty. Ophthalmology. 1993;100:752-761.

29. Newton RH, Meek KM. The integration of the corneal and limbal fibrils in the human eye. Biophys J. 1998;75:2508-2512.

30. Newton RH, Meek KM. Circumcorneal annulus of collagen fibrils in the human limbus. Invest Ophthalmol Vis Sci. 1998;39:1125-1134.

31. Meek KM, Newton RH. Organisation of collagen fibrils in the corneal stroma in relation to mechanical properties and surgical practice. J Refract Surg. 1999;15:695-699. 